\title{
Globally Optimal Synthesis of Heat Exchanger Networks.Part III:Non-isothermal Mixing in Minimal and Non-Minimal Networks
}

\author{
Chenglin Chang ${ }^{1}$, Zuwei Liao ${ }^{1}$, André Costa ${ }^{2}$, and Miguel Bagajewicz ${ }^{3}$ \\ ${ }^{1}$ Zhejiang University \\ ${ }^{2}$ Universidade do Estado do Rio de Janeiro \\ ${ }^{3}$ University of Oklahoma
}

February 16, 2021

\begin{abstract}
In this work, the enumeration algorithms presented in parts I and II for the globally optimal synthesis of heat exchanger networks are extended to consider non-isothermal mixing. The previous models are modified by adding non-isothermal mixing constraints and new models are constructed to target the bounds of the energy consumption and the binding exchanger minimum approximation temperature. These new models are solved using algorithms that involve solving the solution of systems of equations instead of mathematical programming. We also present two alternatives for optimizing each enumerated structure, namely, the use of a global solver, or the use of a golden search with simple resolution of non-isothermal mixing model for fixed energy consumption. The non-isothermal mixing model is reformulated as a convex model, either solved using nonlinear programming or a programming-free methodology, i.e. solving Karush-Kuhn-Tucker equations. A global optimum search algorithm is developed and examples are tested comparing the proposed strategies.
\end{abstract}

\section{Hosted file}

manuscript.pdf available at https://authorea.com/users/396006/articles/509135-globallyoptimal-synthesis-of-heat-exchanger-networks-part-iii-non-isothermal-mixing-in-minimaland-non-minimal-networks 